\title{
What do we do with all the false-positive CA125s?
}

\author{
Scott Wilkes, ${ }^{1}$ Richard Edmondson ${ }^{2}$
}

\begin{abstract}
${ }^{1}$ Honorary Clinical Senior Lecturer, Institute of Health \& Society, Newcastle University, Newcastle upon Tyne, and GP Principal, Coquet Medical Group, Amble Health Centre, Amble, UK ${ }^{2}$ Consultant and Professor of Gynaecological Oncology, Northern Institute of Cancer Research, Newcastle University, Newcastle upon Tyne, and Northern Gynaecological Oncology Centre, Queen Elizabeth Hospital, Gateshead, UK
\end{abstract}

\section{Correspondence to}

Dr Scott Wilkes, Institute of Health \& Society, Newcastle University, Baddiley-Clark Building, Richardson Road, Newcastle upon Tyne NE2 4AX, UK;

scott.wilkes@newcastle.ac.uk

Received 14 January 2013 Revised 18 February 2013 Accepted 20 February 2013 Published Online First 24 May 2013

\section{SLinked}

- http://dx.doi.org/10.1136/ jfprhc-2012-100473

To cite: Wilkes $S$ Edmondson R. J Fam Plann Reprod Health Care 2013;39:160-162.

\section{BACKGROUND}

In their article, Low et al. ${ }^{1}$ have demonstrated a poor awareness of ovarian cancer symptoms among their study population of 1000 UK women. They go on to describe factors associated with delay in help-seeking behaviour of women with symptoms that could be associated with ovarian cancer. These include being too busy to seek help, difficulty making an appointment to see a general practitioner (GP) and a feeling that they may be wasting the GP's time. The challenge for both women and GPs is that the symptoms of early ovarian cancer are either non-existent or non-specific at best and can often mimic the symptoms of many other benign diseases. In day-to-day clinical practice, GPs are faced with multiple non-specific symptoms that can be present in many cancers. A recent analysis of over 23000 cancers in women has led to the development of an algorithm that can indicate the probability of different types of cancer being present to help focus further investigation. ${ }^{2}$ The use of risk assessment tools in general practice is associated with increased investigation, referral and cancer diagnoses. ${ }^{3}$ Currently, most urgent '2-week wait' referral guidelines are symptom-based and target red flag symptoms which are related to latestage diagnosis. ${ }^{45}$ Ovarian cancer is associated with a 5-year survival rate of approximately $35 \%$. The National Institute for Health and Clinical Excellence (NICE) Clinical Guideline CG122 ${ }^{6}$ addresses some of the main problems that are considered to be responsible for the delays that occur in the ovarian cancer pathway, namely detection and diagnosis. Only a minority $(17 \%)$ of ovarian cancers are picked up by GP investigation, with most being diagnosed through routine outpatient referral.

\section{SUMMARY OF NICE CG122}

The key priorities for implementation are largely focused upon GPs. For general practice this can be summarised as follows:

- Investigation of serum CA125 level for women over the age of 50 years who present with one or more persisting symptoms as detailed below.

- Arrange an ultrasound scan of abdomen and pelvis when the CA125 level is $\geq 35 \mathrm{IU} / \mathrm{ml}$.

- For symptomatic women with a CA125 $<35 \mathrm{IU} / \mathrm{ml}$, assess for other causes of their symptoms.

- For symptomatic women with a CA125 $>35 \mathrm{IU} / \mathrm{ml}$ and a normal ultrasound, assess for other causes of their symptoms.

- Calculate a risk of malignancy index (RMI) score (Box 1) and refer all women who have an RMI score $\geq 250$ to specialist gynaecological cancer services.

- A woman presenting with ascites and/or a pelvic or abdominal mass should be referred urgently.

\section{Box 1 Risk of malignancy index I (RMI I)}

RMI I is a product of the ultrasound scan score (U), menopausal status (M) and serum CA125 level.

RMI I $=\mathrm{U} \times \mathrm{M} \times \mathrm{CA} 125$

- The ultrasound result is scored 1 point for each of the following characteristics: multilocular cysts, solid areas, metastases, ascites, bilateral lesions. $\mathrm{U}=0$ for an ultrasound score of 0 points, $U=1$ for an ultrasound score of 1 point, $U=3$ for an ultrasound score of 2-5 points.

- Menopausal status is scored as $1=$ pre-menopausal and $3=$ postmenopausal. The classification of 'post-menopausal' is a woman who has had no period for more than 1 year or a woman aged over 50 years who has had a hysterectomy.

- Serum CA125 is measured in international units per millilitre (IU/ml). 


\section{SYMPTOMS}

Investigating symptomatic women over the age of 50 years is a priority in general practice. Persistent abdominal distension (bloating), feeling full (early satiety), loss of appetite, pelvic or abdominal pain and increased urinary frequency and/or urgency are symptoms that warrant further investigation. ${ }^{6}$ Persistent abdominal distension and loss of appetite have positive predictive values of $2.5 \%$ and $0.6 \%$, respectively, for ovarian cancer. ${ }^{8}$ Whilst these are the best symptom indicators, they are woefully inadequate in predicting the likelihood of having ovarian cancer. Low et al. ${ }^{1}$ have demonstrated that women do not delay help-seeking behaviour when experiencing abdominal pain. However, symptoms of abdominal bloating and loss of appetite were associated with delayed help-seeking behaviour, probably due to misattribution of symptoms rather than a failure to recognise the symptoms.

\section{GP INVESTIGATIONS}

NICE CG122 places a lot of focus on GP assessment of patients and this is particularly crucial because the symptoms of cancer are often the symptoms of benign disease. ${ }^{9}$ Investigations in general practice serve three main functions: first, to identify a cohort of patients that can be reassured that no further intervention is required; second, to identify a cohort of patients that have a problem that can be managed in primary care; and third, to identify a cohort of patients that require onward referral. Depending upon test results, the third group will be referred to secondary/tertiary or private health services. The speed of that referral will subsequently depend upon the potential or actual diagnosis. The investigations that should be carried out on symptomatic women presenting in general practice are determination of serum CA125 level and ultrasound scan of abdomen and pelvis (CG122). ${ }^{6}$ Together these investigations can be used to calculate a RMI score.

\section{RMI INDEX AND GP REFERRAL}

The RMI score is derived from the menopausal status of the women, the serum CA125 and the result of the ultrasound scan (Box 1). The RMI tool has been used by secondary care gynaecologists for many years to determine which patients need to be referred to tertiary gynaecological cancer services for further evaluation. However, with access to ultrasound scanning and serum CA125 measurement, the RMI tool can now be deployed to greater effect in general practice as it has the potential to lead to efficient referral of 'at-risk patients' to specialist gynaecological cancer services.

\section{If the RMI is $>\mathbf{2 5 0}$}

When ovarian cancer is suspected and the RMI score is $\geq 250$, management is straightforward and an urgent '2-week wait' referral is indicated. In some cancer networks this referral may be to the local gynaecologist in secondary care, whereas in other networks referral directly to the gynaecological cancer centre may be advised.

\section{If the RMI is $<\mathbf{2 5 0}$}

Implementation of the RMI index as an assessment of symptomatic women in primary care will generate a significant number of women with abnormal CA125, normal ultrasound scans and therefore RMI scores below the referral threshold. Currently we do not know how many this will be, as data for the symptomatic population are lacking. There are, however, data which show that the situation for asymptomatic women, where a cut-off of $35 \mathrm{IU} / \mathrm{ml}$ is used, will result in a false-positive rate of $2 \%{ }^{10}$ Whilst this may not sound high, the prevalence of ovarian cancer is only 4/10 000, which means that for 10000 women tested with CA125, four will have ovarian cancer and 200 will have a raised CA125 but no ovarian cancer. This false-positive rate increases in pre-menopausal women in whom there is a greater prevalence of the non-malignant causes of a raised CA125 such as endometriosis and fibroids. ${ }^{11}$ Given the non-specificity of symptoms, it is likely that the situation for symptomatic women will not be too different from asymptomatic post-menopausal women.

The NICE guidance is vague on subsequent management of this group of patients, advising: ${ }^{6}$

For any woman who has normal serum CA125 $(<35 \mathrm{IU} / \mathrm{ml})$

or CA125 $\geq 35 \mathrm{IU} / \mathrm{ml}$ but a normal ultrasound:

- assess her carefully for other clinical causes of her symptoms and investigate if appropriate

- if no other clinical cause is apparent, advise her to return to her GP if her symptoms become more frequent and/or persistent.

GPs will therefore need to look for other causes to explain the symptoms and raised CA125. A new diagnosis of irritable bowel syndrome, fibroids or endometriosis in a woman aged over 50 years is unlikely. Other potential causes of raised CA125 include malignancies of the uterus, pancreas, stomach, colon and rectum. Non-malignant causes of raised CA125 include benign ovarian neoplasm, salpingitis, liver cirrhosis, pancreatitis, renal failure and inflammation of mesothelial surfaces including the peritoneum, pleura or pericardium. It is likely that for many of these women with normal ultrasound scans no diagnosis will be achieved, although considerable anxiety may be induced by the knowledge of a raised CA125. It would have been useful if NICE CG122 had included some guidance on what to do in this situation. This may have included an interval for repeating the CA125 measurement and/or ultrasound scan. If symptoms do not resolve and CA125 levels rise over time, this is more indicative of underlying pathology than raised but stable levels. Most women with ovarian 
cancer will demonstrate rapidly rising levels of CA125, in contrast to patients with static or decreasing CA125 who appear to have a very low risk of subsequently developing disease. ${ }^{12}$ How many times and how often measurement of serum CA125 should be repeated is unknown.

\section{WHAT NEXT?}

To date, evidence of implementation of the NICE guideline is lacking. Low et al. ${ }^{1}$ feel that further research is needed to explore the barriers that prevent women with symptoms of abdominal bloating and loss of appetite from seeking help at an early stage, and that evaluation of interventions to increase helpseeking behaviour is required. Evaluation is also required of the impact of false-positive CA125 results and the cost to patients and the National Health Service so that sensible follow-up strategies for women with a raised CA125 but no other evidence of disease can be developed. The potential role of new biomarkers, including human epididymal protein 4 (HE4), are being investigated but so far they do not appear to outperform RMI. ${ }^{13} 14$ There may be potential for panels of biomarkers, yet to be developed, to help improve the specificity and reduce the number of false-positive results.

\section{IN SUMMARY}

It is clear from the study by Low et al. ${ }^{1}$ that there are indeed barriers that prevent women with symptoms associated with ovarian cancer from presenting to GPs. Removing these barriers will lead to a more widespread adoption of the NICE guidance, which in turn will lead to increased use of CA125 in general practice. Although much is known about CA125 and its performance as a biomarker, there are still many unanswered questions regarding its role as a triage tool for women who have a range of relatively nonspecific symptoms. The full impact of the NICE guidance is unclear, but we are concerned that it may identify large numbers of women who have a raised serum CA125 level in the absence of any evidence of other disease. How these women should be managed has not been defined and will need careful thought if we are to avoid generating a cohort of women with high levels of anxiety or who become victims of unnecessary intervention as a result of receiving a false-positive result from a diagnostic test.
Competing interests None.

Provenance and peer review Not commissioned; externally peer reviewed.

\section{REFERENCES}

1 Low E, Waller J, Menon U, et al. Ovarian cancer symptom awareness and anticipated time to help-seeking for symptoms among UK women. J Fam Plann Reprod Health Care 2013:39:163-71.

2 Hippisley-Cox J, Coupland C. Symptoms and risk factors to identify women with suspected cancer in primary care: derivation and validation of an algorithm. Br J Gen Pract 2013;63:16-17.

3 Hamilton W, Green T, Martins T, et al. Evaluation of risk assessment tools for suspected cancer in general practice: a cohort study. Br J Gen Pract 2013;63:20-21.

4 Neal RD, Allgar VL, Ali N, et al. Stage, survival and delays in lung, colorectal, prostate and ovarian cancer: comparison between diagnostic routes. Br J Gen Pract 2007;57:212-219.

5 Jones R, Charlton J, Latinovic R, et al. Alarm symptoms and identification of non-cancer diagnoses in primary care: cohort study. BMJ 2009;339:b3094.

6 National Institute for Health and Clinical Excellence. Ovarian Cancer (CG 122). 2012. http://www.nice.org.uk/cg122 [accessed 12 December 2012].

7 Barrett J, Sharp D, Stapley S, et al. Pathways to the diagnosis of ovarian cancer in the UK: a cohort study in primary care. $\mathrm{Br} \mathrm{J}$ Obstetr Gynaecol 2010;117:1033-1034.

8 Hamilton W, Peters TJ, Bankhead C, et al. Risk of ovarian cancer in women with symptoms in primary care: population based case-control study. BMJ 2009;339:b2998.

9 Hamilton W. Five misconceptions in cancer diagnosis. Br J Gen Pract 2009;59:441-445.

10 Jacobs I, Bast RC Jr. The CA 125 tumour-associated antigen: a review of the literature. Hum Reprod 1994;4:1-12.

11 Skates SJ, Mai P, Horick NK, et al. Large prospective study of ovarian cancer screening in high-risk women: CA125 cut-point defined by menopausal status. Cancer Prev Res 1994;4: 1401-1408.

12 Skates SJ, Menon U, MacDonald N, et al. Calculation of the risk of ovarian cancer from serial CA-125 values for preclinical detection in postmenopausal women. J Clin Oncol 1994; 21(10 Suppl.):206s-210s.

13 Karlsen MA, Sandhu N, Hogdall C, et al. Evaluation of HE4, CA125, risk of ovarian malignancy algorithm (ROMA) and risk of malignancy index (RMI) as diagnostic tools of epithelial ovarian cancer in patients with a pelvic mass. Gynecol Oncol 1994;127:379-383.

14 Van Gorp T, Cadron I, Despierre E, et al. HE4 and CA125 as a diagnostic test in ovarian cancer: prospective validation of the Risk of Ovarian Malignancy Algorithm. Br J Cancer 1994;104:863-870. 